See discussions, stats, and author profiles for this publication at: https://www.researchgate.net/publication/223963261

\title{
Prediction of High-Risk Asymptomatic Carotid Plaques Based on Ultrasonic Image Features
}

Article in IEEE transactions on information technology in biomedicine: a publication of the IEEE Engineering in Medicine and Biology Society · April 2012

DOI: 10.1109/TITB.2012.2192446. Source: PubMed

CITATIONS

30

7 authors, including:

Efthyvoulos C Kyriacou

Frederick University

174 PUBLICATIONS 2,436 CITATIONS

SEE PROFILE

C. S. Pattichis

University of Cyprus

431 PUBLICATIONS 5,663 CITATIONS

SEE PROFILE

Some of the authors of this publication are also working on these related projects:

Deployment of Generic Cross-Boarder eHealth Services in Cyprus View project

Neural Networks in Fetal Medicine View project
Styliani Petroudi University of Cyprus

55 PUBLICATIONS 448 CITATIONS

SEE PROFILE

Marios S Pattichis

University of New Mexico

334 PUBLICATIONS 3,291 CITATIONS

SEE PROFILE 


\title{
Prediction of High-Risk Asymptomatic Carotid Plaques Based on Ultrasonic Image Features
}

\author{
Efthyvoulos C. Kyriacou, Senior Member, IEEE, Styliani Petroudi, Member, IEEE, \\ Constantinos S. Pattichis, Senior Member, IEEE, Marios S. Pattichis, Senior Member, IEEE, Maura Griffin, \\ Stavros Kakkos, and Andrew Nicolaides
}

\begin{abstract}
Carotid plaques have been associated with ipsilateral neurological symptoms. High-resolution ultrasound can provide information not only on the degree of carotid artery stenosis but also on the characteristics of the arterial wall including the size and consistency of atherosclerotic plaques. The aim of this study is to determine whether the addition of ultrasonic plaque texture features to clinical features in patients with asymptomatic internal carotid artery stenosis (ACS) improves the ability to identify plaques that will produce stroke. 1121 patients with ACS have been scanned with ultrasound and followed for a mean of 4 years. It is shown that the combination of texture features based on secondorder statistics spatial gray level dependence matrices (SGLDM) and clinical factors improves stroke prediction (by correctly predicting 89 out of the 108 cases that were symptomatic). Here, the best classification results of $77 \pm 1.8 \%$ were obtained from the use of the SGLDM texture features with support vector machine classifiers. The combination of morphological features with clinical features gave slightly worse classification results of $76 \pm \mathbf{2 . 6 \%}$. These findings need to be further validated in additional prospective studies.
\end{abstract}

Index Terms-Assessment of stroke risk, plaque imaging, ultrasound image analysis.

\section{INTRODUCTION}

A therosclerosis of the internal carotid artery (ICA) is an important risk factor for stroke. Using the North American symptomatic carotid endarterectomy trial method [1] for the determination of stenosis the risk of stroke has been shown to range between $0.1-1.6 \%$ per year for asymptomatic individuals with ICA stenosis $<75-80 \%$. The risk rises to $2-3 \%$

Manuscript received November 3, 2011; revised February 20, 2012; accepted March 19, 2012. Date of publication April 3, 2012; date of current version September 20, 2012.

E. C. Kyriacou is with the Frederick University, Limassol 3080, Cyprus (e-mail: ekyriac@ucy.ac.cy).

S. Petroudi and C. S. Pattichis are with the Department of Computer Science, University of Cyprus, Nicosia CY1678, Cyprus (e-mail: styliani@ucy.ac.cy; pattichi@ucy.ac.cy).

M. S. Pattichis is with the Department of electrical and Computer engineering, University of New Mexico, Albuquerque, NM 87131-0001 USA (e-mail: pattichis@eece.unm.edu).

S. Kakkos is with the Department of Vascular Surgery, University of Patras Medical School, Patras 26504, Greece (e-mail: kakkosstavros@gmail.com).

M. Griffin is with the Vascular Noninvasive Screening and Diagnostic Centre, London, W1G 7BS, U.K. (e-mail: maurabgriffin@ googlemail.com).

A. Nicolaides is with Imperial College London, London, W2 1NY, U.K., with the Vascular Noninvasive Screening and Diagnostic Centre, London, W1G 7BS, U.K., and also with the Cyprus Cardiovascular Disease Educational Research Trust, Nicosia 1678, Cyprus (e-mail: anicolaides1@ gmail.com).

Digital Object Identifier 10.1109/TITB.2012.2192446 per year for individuals with higher grades of stenosis [2]. In the past two decades, carotid endarterectomy has been extensively used for the reduction of stroke risk. This is because two randomized controlled trials, almost a decade apart, the asymptomatic carotid atherosclerosis study in 1995 [3] and the asymptomatic carotid surgery trial in 2004 [4], reported that in patients with asymptomatic ICA stenosis $>60-70 \%$ (using the NASCET method) carotid endarterectomy reduced the risk of stroke from $2 \%$ to $1 \%$ per year [4], [5]. However, in these trials carotid endarterectomy was associated with a $2-3 \%$ perioperative rate of stroke or death, making it marginally effective for asymptomatic patients. It should also be noted that in these trials, medical therapy, which was left to the discretion of the local teams, was suboptimal in relation to current practice. Recent advances in the management of vascular disease and particularly the use of statins appear to have reduced stroke rate to approximately $1 \%$ per year and have prompted doubt regarding the value of endarterectomy in asymptomatic patients [6]-[8]. In addition, medical therapy was found to be three to eight times more cost effective than surgical [9], [10]. However, we believe that carotid endarterectomy can still be justified if subgroups at increased risk can be identified. This raises the need for the establishment of methods for reliable and objective cerebrovascular risk prediction and stratification [2].

High-resolution vascular B mode and Doppler ultrasound provide information not only on the degree of carotid artery stenosis but also on the characteristics of the arterial wall including the size and consistency of atherosclerotic plaques [11]. Carotid stenosis alone has limitations in predicting risk and does not show plaque vulnerability and instability, thus other ultrasonographic plaque morphologic characteristics have been studied for better prediction of risk stroke. Plaque echogenicity as assessed by B-mode ultrasound has been found to reliably predict the content of soft tissue and the amount of calcification in carotid plaques. Additionally, it has been reported that subjects with echolucent atherosclerotic plaques have increased risk of ischemic cerebrovascular events [5]. Most recently, Topakian et al. [12] showed that plaque echolucency can be used to predict stroke. Other studies have reported that plaques that are more echolucent and heterogeneous are often associated with higher cerebrovascular risk and the development of ipsilateral neurological symptoms [2], [13]-[16]. In contrast, homogeneous hypoechoic and hyperechoic plaques without evidence of ulceration usually remain asymptomatic.

Prediction of risk is important as it will aid clinicians in the selection of asymptomatic cases at higher risk. Equally 
important is the establishment of a method that will allow for objective and quantitative evaluation of high-risk cases that are the ones that would most benefit from endarterectomy. This paper tackles exactly this task through presentation of the steps for evaluation of high cerebrovascular risk using a number of clinical factors along with U.S. image analysis for the characterization of plaque morphology and texture.

The combination of clinical and morphological plaque data using first-order statistics for plaque image analysis has already shown the potential of risk stratification in a multicentre cohort study of patients with asymptomatic ICA stenosis called the asymptomatic carotid stenosis and risk of stroke study (ACSRS) [2]. The same images were used in this paper to test the hypothesis that the addition of image analysis techniques using second-order statistics with probabilistic neural networks (PNN) and the support vector machines (SVM) as classifiers, will improve risk stratification.

\section{METHODS}

\section{A. Patient Recruitment}

Newly referred ( $<3$ months) patients with 50-99\% ICA stenosis in relation to the carotid bulb diameter (European carotid surgery trial (ECST) method) without previous ipsilateral cerebral or retinal ischemic (CORI) symptoms, and without neurological abnormalities were recruited to the study after written informed consent. Patients who had had contralateral cerebral hemispheric/retinal or vertebrobasilar symptoms or signs of stroke/TIA were included if asymptomatic for at least six months prior to recruitment. For patients with bilateral carotid atherosclerosis, the side with the more severe stenosis was considered ipsilateral (the study artery) [2].

\section{B. Clinical Characteristics}

At baseline all patients had a history taken and a physical examination by the local neurologist to ensure that they were truly asymptomatic. This examination was repeated at six monthly intervals until a primary endpoint or the end of the study was reached [2]. The major clinical characteristics of the study sample at baseline have already been published in [2]. A subgroup of the main characteristics recorded can be seen in Table I.

\section{Duplex Scanning}

Bilateral carotid duplex scanning was performed on admission to the study. Ultrasonographers from all centers were trained at the coordinating center in grading internal carotid stenosis and plaque image capture. The entire duplex examination, recorded on S-VHS videotape, was sent to the coordinating center for image analysis and quality control.

Images from video recordings were digitized offline on a PC using a video grabber card. Image normalization for gray scale using linear scaling with "blood" (gray scale $=0$ ) and adventitia (gray scale $=190$ ), and pixel density standardization to 20 pixels per mm were performed followed by image analysis. The "Plaque Texture Analysis software" version 3.2 (Iconsoft International Ltd, Greenford, London, U.K.) was used. Plaques
TABLE I

A Subgroup of the Major Clinical CHARACTERISTiCs ReCORded AT BASELINE IN 1121 PATIENTS [2]

\begin{tabular}{|c|c|}
\hline \multicolumn{2}{|c|}{ Continuous variables (normal distribution; mean \pm sd) } \\
\hline Age (years) & $70.0 \pm 7.7$ \\
\hline BMI $\left(\mathrm{kg} / \mathrm{m}^{2}\right)^{*}$ & $25.4 \pm 3.7$ \\
\hline $\mathrm{SBP}(\mathrm{mmHg}) *$ & $152.3 \pm 22.2$ \\
\hline $\mathrm{DBP}(\mathrm{mmHg})^{*}$ & $82.2 \pm 10.0$ \\
\hline Total Cholesterol (mmol/dL)* & $6.01 \pm 1.19$ \\
\hline LDL Cholesterol (mmol/dL)* & $3.90 \pm 1.20$ \\
\hline HDL Cholesterol (mmol/dL)* & $1.30 \pm 0.49$ \\
\hline Creatinine $(\mu \mathrm{mol} / \mathrm{L})^{*}$ & $100.5 \pm 34.1$ \\
\hline \multicolumn{2}{|c|}{$\begin{array}{l}\text { Continuous variables (skewed distribution; median, interquartile } \\
\text { range) }\end{array}$} \\
\hline Pack-years & $10(0,36)$ \\
\hline Triglycerides $(\mathrm{mmol} / \mathrm{dL})^{*}$ & $1.58(1.17,2.19)$ \\
\hline \multicolumn{2}{|l|}{ Categorical variables } \\
\hline Hypertension & $709(63 \%)$ \\
\hline Diabetes & $231(21 \%)$ \\
\hline Coronary artery disease & $379(34 \%)$ \\
\hline \multicolumn{2}{|c|}{$\begin{array}{l}\text { * Percentages of missing values were BMI: } 4 \% \text {, SBP: } 10 \% \text {, DBP: } 10 \% \text {, } \\
\text { creatinine: } 11 \% \text {, fibrinogen: } 23 \% \text {, hematocrit: } 12 \% \text {, total cholesterol: } 11 \% \text {, } \\
\text { LDL: } 22 \% \text {, HDL: } 22 \% \text {, triglycerides: } 12 \% \text {. Percentages of baseline treatment } \\
80 \% \text { of patients were using statins and } 20 \% \text { aspirin. }\end{array}$} \\
\hline
\end{tabular}

were segmented by expert physicians using the same software [2], [17].

\section{Texture Features}

Several image texture analysis features were extracted and used in the statistical analysis [18], [19]. These features have been presented in previous studies and have been computed using the "Plaque Texture Analysis software" following image normalization carried out as documented in the previous subsection. The algorithms used for this study namely are 1) statistical features [18], and 2) SGLDM features [18].

Morphological features associated with plaque composition as described in [19] were also considered. This led to the consideration of morphological features that come from 1) dark regions associated with lipid, thrombus, blood, or haemorrhage, 2) bright regions associated with collagen and calcified components, and 3) medium-brightness regions that fall between them. As discussed in [19], the most promising results were given by morphological analysis of the dark image components. Following image normalization, the binary $L$ images are generated using

$$
L=\{(i, j): \text { such that } I(i, j)<25\}
$$

where $I$ denotes the normalized image. A multiscale morphological decomposition of each binary image is generated using the difference images

$$
\begin{aligned}
d_{0}(L ; B) & =L-L \circ B \\
d_{1}(L ; B) & =L \circ B-L \circ 2 B \\
\vdots & \\
d_{n-1}(L ; B) & =L \circ(n-1) B-L \circ n B
\end{aligned}
$$

where $B$ denotes the " + " structural element, o denotes the morphological open operation, and $d_{n}$ denotes the binary difference image. The binary difference images are then used to generate 
the morphological probability density function (pdf) using

$$
\operatorname{pdf}_{K}(n, B)=\frac{A\left(d_{n}(L ; B)\right)}{A(L)}
$$

where $A(L)$ represents the number of pixels in the image, and $n$ was allowed to vary from 1 to 70 . The cumulative distribution function (cdf) can be constructed using pdf:

$$
\operatorname{cdf}_{L}(n, B)= \begin{cases}0, & n=0 \\ \sum_{r=0}^{n-1} \operatorname{pdf}_{L}(r, B), & n>0\end{cases}
$$

Images that gave significant differences in the classification of symptomatic versus asymptomatic cases were used as texture features. This led to the use of

$$
\operatorname{pdf}_{L}(r, B), \operatorname{cdf}(p, B), \quad r, p=1, \ldots, 5
$$

as explained in [19].

\section{E. Risk Modeling}

Risk modeling was carried out using two different classifiers: the SVM, and the PNN. These classifiers were trained to classify the feature sets (FS) investigated into two classes: 1) asymptomatic plaques or 2) stroke (including TIAs) (or symptomatic) plaques, i.e., unstable plaques.

The SVM method is initially based on a nonlinear mapping of the initial dataset using a function $\varphi($.$) , and then the identifi-$ cation of a hyperplane, which is able to achieve the separation of two categories of data. The vectors defining the hyperplanes can be chosen to be linear combinations with parameters $\alpha_{i}$ of images of feature vectors that occur in the database. With this choice of a hyperplane, the points $x$ in the feature space that are mapped into the hyperplane are defined by the relation

$$
\sum \alpha_{i} K\left(x_{i}, x\right)=\text { constant. }
$$

If $K(x, y)$ becomes small as $y$ grows further from $x$, each element in the sum measures the degree of closeness of the test point $x$ to the corresponding database point $x_{i}$. In this way, the sum of kernels as mentioned earlier can be used to measure the relative nearness of each test point to the data points originating in one or the other of the sets to be discriminated.

Details about the implementation of the SVM algorithm used can be found in [20] and [21]. The SVM network was investigated using Gaussian radial basis function (RBF) kernels; this was decided as the rest of the kernel functions could not achieve satisfactory results. The SVM with RBF kernel was investigated using tenfold cross validation in order to identify the best parameters such as the spread of.

The PNN [22] classifier was used for developing classification models for the problem under study. The PNN falls within the category of nearest-neighbor classifiers. For a given vector $\mathbf{w}$ to be classified, an activation $a_{i}$ is computed for each of the two classes of plaques $(i=1, \ldots, 2)$. The activation $a_{i}$ is defined to be the total distance of $\mathbf{w}$ from each of the $M_{i}$ prototype feature vectors $\mathbf{x}_{j}^{(i)}$ that belong to the $i$ th class

$$
a_{i}=\sum_{j=1}^{M_{i}} \exp \left[-\beta\left(\mathbf{w}-\mathbf{x}_{j}^{(i)}\right)^{T}\left(\mathbf{w}-\mathbf{x}_{j}^{(i)}\right)\right]
$$

TABLE II

NUMBER OF ASYMPTOMATIC AND STROKE (INCLUDING TIAS) CASES, SEPARATED INTO SUBGROUPS ACCORDING TO ECST STENOSIS AND NASCET STENOSIS

\begin{tabular}{lclll}
\hline $\begin{array}{l}\text { ECST } \\
\text { stenosis(\%) }\end{array}$ & $\begin{array}{l}\text { NASCET } \\
\text { stenosis(\%) }\end{array}$ & No. & Asymptomatic & Stroke \\
\hline All patients & & 1121 & $991(88.4 \%)$ & $108(9.6 \%)$ \\
$50-69$ & $<50$ & 198 & $182(92.0 \%)$ & $12(6.1 \%)$ \\
$70-89$ & $50-82$ & 598 & $533(89.1 \%)$ & $56(9.4 \%)$ \\
$90-99$ & $83-99$ & 325 & $276(84.9 \%)$ & $40(12.3 \%)$ \\
\hline Follow-up 6 months to 8 years (mean, 48 months) [2]. \\
TIAs: transient ischemic attacks; \\
ECST: European carotid surgery trial; \\
NASCET: North American symptomatic carotid endarterectomy trial.
\end{tabular}

where $\beta$ is a smoothing factor. This classifier was investigated for several spread radii in order to identify the best radius for the current problem.

\section{F. Evaluation}

The performances of the classifier systems were measured using the following parameters: 1) true positives when the system correctly classifies plaques as symptomatic, 2) false positives (FP) where the system wrongly classifies plaques symptomatic while they are asymptomatic, 3) false negatives when the system wrongly classifies plaques as asymptomatic while they are symptomatic, and 4) true negatives when the system correctly classifies plaques as asymptomatic. To evaluate the ability of the classifiers to predict high-risk cases the sensitivity (SE), which is the likelihood that a symptomatic plaque will be detected given that it is symptomatic, and specificity (SP) which is the likelihood that a plaque will be classified as asymptomatic given that it is asymptomatic, were also evaluated. For the overall performance, the correct classification (CC) rate, which gives the percentage of correctly classified plaques, is also provided.

\section{RESULTS}

\section{A. Dataset Investigated}

A total of 1121 patients between 39 and 89 years (mean age $70.0 \pm \mathrm{SD} 7.7,61 \%$ male) were recruited during 19982002 with a follow-up of 6-96 months (mean 48 months) in the context of the ACSRS study (see Table II). A total of 130 first ipsilateral CORI events occurred (59 strokes of which 12 were fatal, 49 TIAs and 22 amaurosis fugax). There were 49 first contralateral CORI events (18 ischemic strokes of which seven were fatal, 22 TIAs and nine amaurosis fugax). There were two vertebrobasilar strokes. AF cases were excluded from the study, thus having 1099 cases.

There have been a total of 214 deaths (195 nonstroke deaths) of which 157 (73\%) were due to cardiovascular causes: myocardial infarction-110, fatal stroke-19 (12 ipsilateral and 7 contralateral already mentioned earlier), heart failure-17, pulmonary embolism-3, lower limb ischemia/gangrene-3, ruptured abdominal aortic aneurysm-3, renal failure - 1 , and mesenteric artery thrombosis-1. There were 56 nonvascular deaths; malignancy: 37 , pneumonia/respiratory failure: 12 , gastrointestinal hemorrhage: 2 , dementia: 2 , road traffic accident: 2 
and general surgical procedure: 1. Cause of death was unknown in one patient.

\section{B. Feature Sets Extracted}

The following FS were extracted from the asymptomatic and stroke (including TIAs) cases given in Table II.

1) FS 1: ACSRS clinical and plaque features [2]: stenosis $(\% \mathrm{ECST}), \log (\mathrm{GSM}+40)$, (plaque area $)^{1 / 3}$, discrete white areas (DWA) absent or present, and history of contralateral TIAs and/or stroke.

2) FS 2: texture features-SGLDM [18]: Spatial Gray Level Dependence Matrices; ASM: Angular Second Moment; CON: Contrast; SAV: Sum Average, SVAR: Sum Variance; ENT: Entropy; Diffvar: Difference Variance.

3) FS 3: Texture Features-Morphology [19]: Low image cdf $1-5$ and pdf $1-5$.

These FS are tabulated in Table III together with the results of univariate analysis. The $\mathrm{p}$ value using $t$-test for the continuous variables and chi-square test for the categorical variables (DWA, Hist TIAs). Features with significant difference at $\mathrm{P}<0.001$ were used in the prediction models. Fig. 1 shows examples of normalized gray-scale and color-contoured images of segmented plaques with selected plaque features for both asymptomatic and symptomatic plaques.

It is noted that as documented in the ACSRS study [2], comorbid conditions such as LDL, diabetes, etc., were not associated with the development of stroke in this population because they were high or present in the majority of patients. Also, the population of patients have $>50 \%$ carotid stenosis and are all high risk arteriopaths.

Compared to the clinical features, it is interesting to note that the mean values for the different texture features give relatively large differences between asymptomatic and stroke cases. Unfortunately, texture features also exhibit higher variations than clinical features.

\section{Risk Modeling}

High risk modeling using the prediction results of asymptomatic versus stroke (including TIAs) are given in Table IV based on the FS tabulated in Table III-FS 1: ACSRS clinical and plaque features; FS 2: texture features-SGLDM; FS 3: texture features-morphology. Risk modeling was carried out using the probabilistic neural networks $(\mathrm{P})$ and the support vector machines (S) classification models. The results are the average of 2000 runs, where 10 bootstrapping sets of 100 asymptomatic and 100 stroke (including TIAs) cases were drawn at random from the corresponding 991 asymptomatic, and 108 stroke cases (as tabulated in Table II); the performance of each one of this sets was then evaluated using the leave one out method.

The best classification results of $77 \pm 1.8 \%$ were obtained from the use of the SGLDM texture features with SVM classifiers. The combination of morphological features with clinical features gave slightly worse classification results of $76 \pm 2.6 \%$.

All the PNN models gave poorer prediction performance as shown in Table IV. Moreover, it should be noted that it has been found that there is significant difference (at $P<0.01$ level) when
TABLE III

LIST OF RISK FACTORS-FS INVESTIGATED FOR THE DATASETS GIVEN IN TABLE II FOR THE ASYMPTOMATIC AND STROKE (INCLUDING TIAS) PLAQUES

\begin{tabular}{|c|c|c|c|}
\hline & 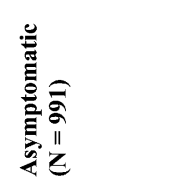 & 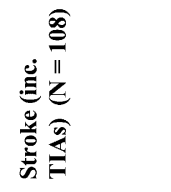 & 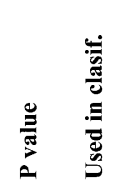 \\
\hline \multicolumn{4}{|c|}{ FS 1: ACSRS Clinical and plaque features [2] } \\
\hline Stenosis $(\% \mathrm{ECST})$ & $77.64 \pm 12.830$ & $81.52 \pm 11.673$ & 0.002 yes \\
\hline $\log (G S M+40)$ & $4.301 \pm .313$ & $4.058 \pm .250$ & 0.000 yes \\
\hline 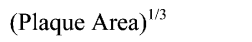 & $3.40 \pm .676$ & $3.987 \pm .794$ & 0.000 yes \\
\hline DWAs (\#of Yes cases) & 614 & 88 & 0.000 yes \\
\hline $\begin{array}{l}\text { History of contr. TIAs } \\
\text { and/or Stroke (\#of Yes } \\
\text { cases) }\end{array}$ & 139 & 34 & 0.000 yes \\
\hline \multicolumn{4}{|c|}{ FS 2: Texture Features - SGLDM [19] } \\
\hline SGLDM_ASM_mean & $4.42 \pm .738$ & $3.92 \pm .838$ & 0.000 yes \\
\hline SGLDM_con_mean & $.026 \pm 0.0802$ & $.0676 \pm .1150$ & 0.000 yes \\
\hline $\ln ($ SGLDM_con_mean) & $-5.813 \pm 1.8080$ & $-4.018 \pm 1.7830$ & 0.000 yes \\
\hline SGLDM_SAV_range & $.306 \pm .1341$ & $.412 \pm .1482$ & 0.000 yes \\
\hline SGLDM_SVAR_mean & $94.692 \pm 49.2841$ & $\begin{array}{l}61.211 \pm \\
33.3339\end{array}$ & 0.000 yes \\
\hline SGLDM_ent_mean & $5.018 \pm 0.8032$ & $4.512 \pm 0.9301$ & 0.000 yes \\
\hline ln(SGLDM_ent_mean) & $2.245 \pm .1992$ & $2.114 \pm .2382$ & 0.000 yes \\
\hline SGLDM_diffvar_mean & $7.0951 \pm 1.2341$ & $6.220 \pm 1.3939$ & 0.000 yes \\
\hline \multicolumn{4}{|c|}{ FS 3: Texture Features - Morphology [20] } \\
\hline $\mathrm{L}$ image $\operatorname{cdf}$ for $\mathrm{n}=1$ & $.0438 \pm .0451$ & $.019 \pm .0218$ & 0.000 yes \\
\hline $\mathrm{L}$ image $\operatorname{cdf}$ for $\mathrm{n}=2$ & $.120 \pm .1063$ & $.057 \pm .0611$ & 0.000 yes \\
\hline L image cdf for $n=3$ & $.208 \pm .1641$ & $.103 \pm .1054$ & 0.000 yes \\
\hline $\mathrm{L}$ image $\operatorname{cdf}$ for $\mathrm{n}=4$ & $.294 \pm .2110$ & $.148 \pm .1358$ & 0.000 yes \\
\hline L image cdf for $n=5$ & $.374 \pm .2491$ & $.193 \pm .1628$ & 0.000 yes \\
\hline $\mathrm{L}$ image pdf for $\mathrm{n}=1$ & $.0438 \pm .0451$ & $.019 \pm .0218$ & 0.000 yes \\
\hline $\mathrm{L}$ image pdf for $\mathrm{n}=2$ & $.076 \pm .0646$ & $.037 \pm .0100$ & 0.000 yes \\
\hline$L$ image pdf for $n=3$ & $.088 \pm .0662$ & $.047 \pm .0475$ & 0.000 yes \\
\hline$L$ image pdf for $n=4$ & $.086 \pm .0609$ & $.044 \pm .0330$ & 0.000 yes \\
\hline L image pdf for $n=5$ & $.080 \pm .0563$ & $.045 \pm .0331$ & 0.000 yes \\
\hline \multicolumn{4}{|c|}{$\begin{array}{l}\text { For the continuous variables, mean } \pm \text { standard deviation, and for categorical } \\
\text { variables the frequency of yes/no are tabulated. Univariate analysis (t-test) was } \\
\text { carried out for the continuous parameters and the P value is given, and chi-square } \\
\text { test for the categorical parameters. Features that are significantly different at } \\
\mathrm{P}<0.001 \text { were used for the risk modeling as given in the last column. TIAs: } \\
\text { transient ischemic attacks; ECST: European carotid surgery trial; GSM: } \\
\text { gray-scale median; DWA: discrete white areas (present orabsent); SGLDM: } \\
\text { spatial gray level dependence matrices---ASM: angular second moment, CON: } \\
\text { contrast, SAV: sum average, SVAR: sum variance, ENT: entropy, Diffvar: } \\
\text { difference variance. }\end{array}$} \\
\hline
\end{tabular}

comparing the SE and SP measures using the chi-square test for SVM versus PNN risk-prediction models for all FS.

Table $\mathrm{V}$ tabulates the results of the best risk prediction model based on SVM using the SGLDM plaque texture features. It can be seen that for this model, for the 1099 cases from the ACSRS dataset, the SVM model could correctly predict at baseline 89 out of the 108 cases that were symptomatic.

It is noted that the area under the Receiver Operated Characteristics (ROC) curve for model FS1 was 0.832 (95\% CI 0.808 to 0.853 ), for FS2 was 0.803 (95\% CI 0.778 to 0.826 ), and for FS3 was 0.571 (95\% CI 0.539 to 0.602 ), whereas the ROC for stenosis alone in predicting stroke was 0.59 (95\% CI 0.563 to $0.623)$. 


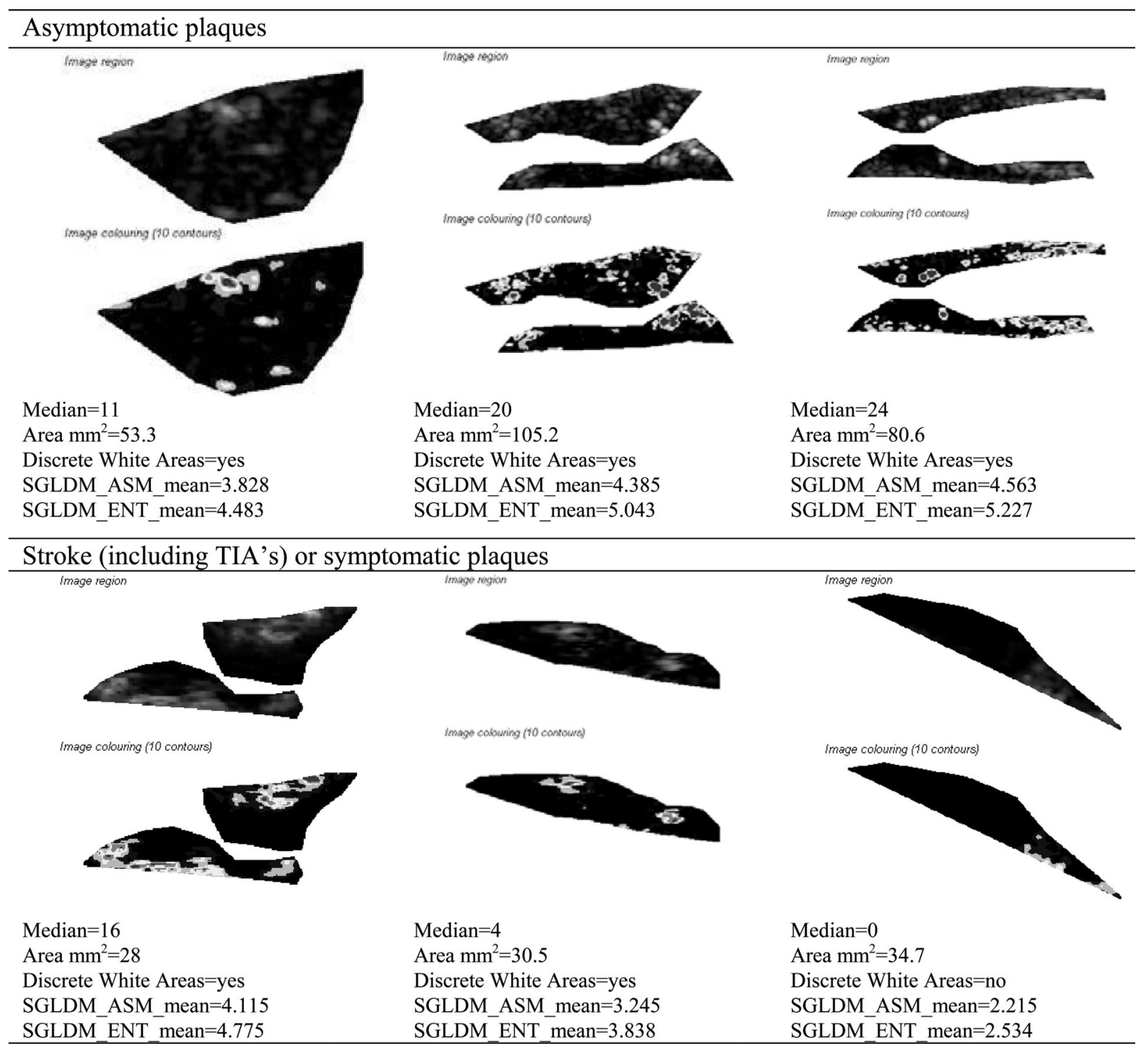

Fig. 1. Examples of normalized gray-scale and color-contoured images of segmented plaques with selected plaque features: (a) asymptomatic, and (b) stroke (including TIAs) or symptomatic (see Table II for feature description).

\section{DISCUSSION}

Cerebrovascular risk in asymptomatic patients has been shown to depend on the degree of stenosis, clinical features and the presence, morphology and texture of carotid plaque as assessed by ultrasound [2]. However, visual evaluation of the morphology and texture of the carotid plaque is difficult and can be enhanced with the use of image analysis methods [12], [23]-[25]. Griffin et al. [23] showed that the use of image normalization resulted in reclassification of the visually evaluated types of plaques associated with a high cerebrovascular event for asymptomatic cases. Visual assessment of the carotid plaque can offer qualitative information about tissue characterization but without precision and with a moderate-togood agreement between and within-observers, is subjective and operator dependent [26].
The evaluation of high-risk asymptomatic cases for the cerebrovascular disease is based on a significant number of different determinants that interact in complex and nonlinear ways. The use of computer aided image classification enables better quantification and standardization of the predicted risk. In addition the use of computer-aided analysis provides for objective, reproducible and quantitative determinants on which this analysis can be based. In previous work different texture and morphology measures for plaque characterization have been developed and validated [19]. These determinants along with clinical features have been used for the development of cerebrovascular statistical risk stratification prediction models [2].

Neural networks compared to statistical models are more complex and can better characterize complex nonlinear relationships amongst the different determinants and may produce a model of greater discriminatory power and accuracy as they 
TABLE IV

High-Risk MOdELING PREDICTION RESUlts OF ASYMPTOMATIC VERSUS STROKE (INCLUding TIAs), MEASURES (MEAN \pm STANDARD DEVIATION) ARE GIVEN FOR 2000 RUNS (10 BOOTSTRAPPING SETS OF 100 ASYMPTOMATIC AND 100 STROKE (INCLUDING TIAS) CASES DRAWN AT RANDOM FROM THE CORRESPONDING 991 ASYMPTOMATIC, AND 108 STROKE CASES; AND THE LEAVE ONE OUT METHOD WAS THEN RUN ON EACH OF THE 200 SET)

\begin{tabular}{|c|c|c|c|c|c|c|c|c|}
\hline 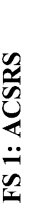 & 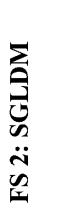 & 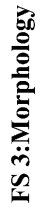 & 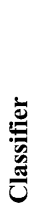 & 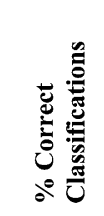 & 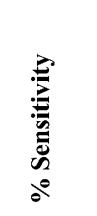 & 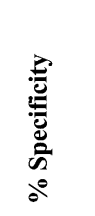 & 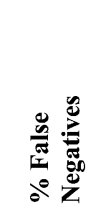 & 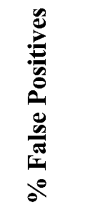 \\
\hline+ & & & $\mathrm{S}$ & $74 \pm 2.5$ & $78 \pm 3.8$ & $71 \pm 4.0$ & $23 \pm 3.8$ & $30 \pm 4.0$ \\
\hline \multirow[t]{5}{*}{+} & & & $\mathrm{P}$ & $64 \pm 4.2$ & $64 \pm 3.3$ & $65 \pm 5.3$ & $36 \pm 3.3$ & $35 \pm 5.3$ \\
\hline & + & & $\mathrm{S}$ & $77 \pm 1.8$ & $82 \pm 3.4$ & $72 \pm 1.2$ & $18 \pm 3.4$ & $28 \pm 1.2$ \\
\hline & + & & $\mathrm{P}$ & $65 \pm 4.8$ & $65 \pm 6$ & $65 \pm 4.6$ & $35 \pm 6$ & $35 \pm 4.6$ \\
\hline & & + & $\mathrm{S}$ & $72 \pm 1.8$ & $82 \pm 4.3$ & $63 \pm 3.2$ & $18 \pm 4.3$ & $37 \pm 3.2$ \\
\hline & & + & $\mathrm{P}$ & $63 \pm 2.2$ & $70 \pm 3.6$ & $56 \pm 4.6$ & $30 \pm 3.6$ & $44 \pm 4.6$ \\
\hline+ & + & & $\mathrm{S}$ & $76 \pm 2.4$ & $79 \pm 3.5$ & $72 \pm 3.0$ & $21 \pm 3.5$ & $28 \pm 3.0$ \\
\hline+ & + & & $\mathrm{P}$ & $64 \pm 2.9$ & $63 \pm 4.9$ & $66 \pm 3.2$ & $37 \pm 4.9$ & $34 \pm 3.2$ \\
\hline+ & & + & $\mathrm{S}$ & $76 \pm 2.6$ & $80 \pm 5$ & $71 \pm 6.3$ & $20 \pm 5$ & $29 \pm 6.3$ \\
\hline \multirow[t]{3}{*}{+} & & + & $\mathrm{P}$ & $67 \pm 2.9$ & $69 \pm 3.9$ & $64 \pm 3.2$ & $31 \pm 3.9$ & $36 \pm 3.2$ \\
\hline & + & + & $\mathrm{S}$ & $74 \pm 3.5$ & $78 \pm 4.7$ & $70 \pm 9.7$ & $22 \pm 4.7$ & $30 \pm 9.7$ \\
\hline & + & + & $\mathrm{P}$ & $65 \pm 3.9$ & $65 \pm 5.9$ & $65 \pm 3.2$ & $35 \pm 5.9$ & $35 \pm 3.2$ \\
\hline+ & + & + & S & $75 \pm 4.2$ & $78 \pm 5.0$ & $73 \pm 6.9$ & $22 \pm 5.0$ & $27 \pm 6.9$ \\
\hline+ & + & + & $\mathrm{P}$ & $64 \pm 3.5$ & $63 \pm 4.2$ & $66 \pm 4.2$ & $37 \pm 4.2$ & $34 \pm 4.2$ \\
\hline
\end{tabular}

TABLE V

CONFUSION MATRICES FOR THE BEST SVM PREDICTION MODEL AND CORRESPONDING PNN MODEL BASED ON FS2 TEXTURE-SGLDM FEATURES

\begin{tabular}{|c|c|c|c|c|}
\hline & \multicolumn{4}{|c|}{ Predicted } \\
\hline \multirow[b]{2}{*}{ Observed } & SI & $V M$ & $\mathbf{P I}$ & $\mathbf{N N}$ \\
\hline & Sym. & Asym. & Sym. & Asym. \\
\hline Sym. 108 & $89(82 \%)$ & $19(18 \%)$ & $71(65 \%)$ & $37(35 \%)$ \\
\hline Asym. 991 & $277(28 \%)$ & $714(72 \%)$ & $346(35 \%)$ & $645(65 \%)$ \\
\hline Total 1099 & 366 & 733 & 417 & 682 \\
\hline \multicolumn{5}{|c|}{$\begin{array}{l}\text { Sym. represents high-risk cases that ended up having a stroke (including TIAs), } \\
\text { whereas Asym. represents asymptomatic cases. AF cases were excluded from } \\
\text { the study. } \\
\text { Positive predictive value (PPV) }=24 \% \text { (SVM), } 17 \% \text { (PNN). } \\
\text { Negative predictive value (NPV) }=97 \% \text { (SVM), } 94.5 \% \text { (PNN). } \\
\text { Correct classifications }=73 \% \text { (SVM), } 65 \% \text { (PNN). }\end{array}$} \\
\hline
\end{tabular}

create a functional form and fit the data at the same time [27]. In this study two different types of neural networks were used for the classification of asymptomatic cases to high and low risk for suffering a stroke based on the aforementioned determinants, as different types of neural networks may perform differently depending on the clinical applications [28]. Both PNNs and SVMs were applied as their use has been investigated in the classification of morphological features of carotid atherosclerotic plaque in a cross-sectional study [19]. SVMs were consistently superior to PNNs as in [19]. SVM is an efficient classification method, which has yielded superior performance compared to regression and other prediction [29]. In addition SVMs compared to other classifiers, aim at minimizing a bound on the generalization error, instead of simply minimizing the training error and are less affected by the dimensionality of the problem, but rather on the "margin" which separates the data [30].

In the present study, the neural networks were extensively evaluated on different determinants and combinations thereof, including the ACSRS clinical and plaque texture and morphology features [2]. For the evaluation, the accuracy in classification along with the SE and SP, and the corresponding positive predictive values and negative predictive values were evaluated for different combination of the three different groups of features for the prediction of stroke as shown in Tables IV and V. The best classification - which is achieved using the SGLDM plaque texture features-allows for a correct prediction of $77 \pm 1.8$ of the cases that will either develop symptoms or not, with a SE of $82 \pm 3.4$ and a SP of $72 \pm 1.2$ as shown in Table V. This according to the risk evaluated here means that $82 \%$ of the cases that would result in TIA or stroke can be predicted with only $28 \%$ FP.

The value of the corresponding classification lies in its ability to identify the individuals who are at high risk of having a stroke and who, therefore, may require invasive intervention whilst excluding those who do not require such intervention and medical treatment alone can allow for lower stroke risk.

It should, however, be noted that there are certain limitations of the risk processing proposed in the current study as a result of the investigated dataset. These limitations are the following. 1) The importance of accurate prediction is greater in the "gray zone" with degree of ECST stenosis between 70\% and $89 \%$, (number of subjects $=598$, and number of strokes $=56$, from Table II) where surgery is not considered always mandatory. However, subgroup analysis was not carried out because it would have weakened the statistical power because of the small number of strokes. Stenosis has been included as a covariable in the clinical model FS1. Therefore, the results apply to all patients irrespective of degree of stenosis. When stenosis is added to the other models, it is no longer significant. 2) Prognosis of moderate-severe carotid stenosis is markedly improved in the last ten years in medically treated patients with optimal therapy. Since in ACSRS only $25 \%$ of patients were treated at baseline with lipid lowering therapy (although these rates markedly increased in the final phase of the study), this feature could not be correctly employed in the predictive models. 3) Another limitation is related to the duration of the follow-up of the cases investigated. More specifically, there were 108 ipsilateral ictus or TIA events that were compared with 991 "asymptomatic cases" for definition of the risk model. However, study exit points were all deaths different from ipsilateral stroke (contralateral: 7 deaths, nonstroke deaths: 214), and carotid revascularization. Thus, more than $25 \%$ of asymptomatic cases had a fatal event during the follow-up. Whether the occurrence of event resulted in a shorter follow-up duration with respect to the remaining patients has not been analyzed. Shorter observation times of this population might have affected the overall results of SVM and PNN analyses.

As it has been noted in the introduction, the high prevalence of patients with asymptomatic carotid artery stenosis had lead to the extensive use of endarterectomy and overtreatment, the benefits of which have been shown to be absorbed by receiving 
medical intervention alone, if all asymptomatic cases are considered. This has resulted in blurring of the distinction of when endarterectomy for stroke prevention should be used. However, when higher risk patients are considered, medical intervention alone may result in undertreatment and endarterectomy may be more appropriate for risk reduction.

The need to target more invasive treatment toward high risk for stroke asymptomatic patients cannot be overstated, as the cost of screening and intervention will not only continue to grow but will draw from other cost-effective preventive strategies [31]. This is especially true for cerebrovascular disease as recent studies have shown medical intervention alone may be expected to reduce stroke risk to approximately $1 \%$. By using the SVM classification on the plaque texture features and translating the results of the presented method to the ACSRS dataset can mean that out of the asymptomatic 108 cases that would result in stroke or TIA 89 could have been predicted at the baseline as shown in Table V. Furthermore, for the SVM classifier using the plaque SGLDM texture features, incorporating the total cost analysis as presented by McPhee et al. [32], and assuming all ACSRS cases being treated with endarterectomy for stroke prevention, it can be estimated that a total of $\$ 19.825$ million $(1121 \times \$ 17685)$ would be spend and 991 unnecessary invasive procedures would be performed for the prevention of 108 strokes (assuming no procedural risk). The cost incurred, in this scenario, for preventing one stroke in eight years would, therefore, be almost $\$ 184$ thousands. It should be noted that this number is estimated on a study, where the patients were undergoing medical intervention and more than half of the cases $(607 / 1121)$ exhibited stenosis over $80 \%$ and correspond to a group where intervention is considered imperative [33]. According to McPhee et al. [32], 122986 invasive revascularizations in asymptomatic patients were performed in the U.S. in 2005. Naylor et al. [31], using the ACAS [3] data, as in [32], showed 122986 invasive procedures would be performed to prevent 7256 strokes-with 115730 unnecessary procedures - so that the cost to prevent an ipsilateral stroke would reach $\$ 319$ thousand, at a total cost of $\$ 2.1$ billion. Extending the achieved high risk for stroke classification to the U.S. statistics [32] and taking no other medical treatment or risk into account, the presented method could allow for better justification of appropriate prevention in 5950 cases (out of the 7256) with only 32 404 — compared to the 122986 unnecessary invasive interventions, at the baseline, and at a total cost of $\$ 679$ million compared to $\$ 2.1$ billion, and a cost of $\$ 114$ thousand per prevented stroke.

\section{CONCLUDing REMARKS}

The aim of this paper has been to determine whether computed aided image analysis provides for better objective and quantitative features for the prediction of stroke risk that could lead to the development of computer aided stroke risk prediction systems. Such systems will allow for objective and quantitative prediction of the high-risk cases for stroke. The prediction results achieved using the SVM classifier are very encouraging. However, as the plaque features are all developed and tested on the same cohort study; in the future, they need to be tested on another prospective study. Also, the validation of predicted risk should be performed in a separate cohort of patients, with or without cerebral events in the follow-up, with comparable follow-up duration.

To the best of our knowledge, this is the first report that uses neural networks and a combination of clinical and computer aided plaque morphology and texture measures to calculate the risk of stroke in a prospective study. The results suggest that the clinical and plaque characterization determinants can be used to better evaluate and choose the different treatment options for the prevention of cerebrovascular disease, and to differentiate between high risk and asymptomatic subjects.

\section{REFERENCES}

[1] A. Fox, "How to measure carotid stenosis," Radiology, vol. 186, pp. 316 318, 1993.

[2] A. Nicolaides, S. Kakkos, E. Kyriacou, M. Griffin, M. Sabetai, D. J. Thomas, T. Tegos, G. Geroulakos, N. Labropoulos, C. J. Doré, T. P. Morris, R. Naylor, and A. L. Abbott, "Asymptomatic internal carotid artery stenosis and cerebrovascular risk stratification," J. Vasc. Surg., vol. 52, no. 4, pp. 1486-1496, Dec. 2010.

[3] Executive Committee for the Asymptomatic Carotid Atherosclerosis Study, "Endarterectomy for asymptomatic carotid artery stenosis," J. Amer. Med. Assoc., vol. 273, pp. 1421-1428, 1995.

[4] MRC Asymptomatic Carotid Surgery Trial (ACST) Collaborative Group, "Prevention of disabling and fatal strokes by successful carotid endarterectomy in patients without recent neurological symptoms: Randomized controlled trial," Lancet, vol. 363, pp. 1491-502, 2004.

[5] E. Mathiesen, K. Bonan, and O. Joakimsen, "Echolucent plaques are associated with high risk of ischemic cerebrovascular events in carotid stenosis: The tromso study," Circulation, vol. 103, pp. 2171-2175, 2001.

[6] A. L. Abbott and G. A. Donnan, "Does the 'High Risk' patient with asymptomatic carotid stenosis really exist?," Eur. J. Endovasc. Surg., vol. 35, pp. 524-533, 2008.

[7] A. R. Naylor, P. A. Gaines, and P. M. Rothwell, "Who benefits most from intervention in asymptomatic carotid stenosis: Patients or professionals?," Eur. J. Vasc. Endovasc. Surg., vol. 37, pp. 625-632, 2009.

[8] A. L. Abbott, "Medical (nonsurgical) intervention alone is now best for prevention of stroke associated with asymptomatic severe carotid stenosis: Results of a systematic review and analysis," Stroke, vol. 40, pp. 573-583, 2009.

[9] A. Abbott, "Asympotmatic carotid artery stenosis-it's time to stop operating," Nature Clin. Practice Neurol., vol. 4, pp. 4-5, 2008.

[10] A. Abbott, "Medical (Nonsurgical) intervention alone is now best for prevention of stroke associated with asympotmatic severe carotid stenosis: Results of a systematic review and analysis," Stroke, vol. 40, pp. 573-583, 2009.

[11] M. Reiter, R. Horvat, S. Puchner, W. Rinner, P. Polterauer, J. Lammer, E. Minar, and R. A. Bucek, "Plaque imaging of the internal carotid artery-Correlation of B-flow imaging with histopathology," Amer. J. Neuroradiol., vol. 28, pp. 122-126, 2007.

[12] R. Topakian, A. King, S. Kwon, A. Schaafsma, M. Shipley, and H. Markus, "Ultrasonic plaque echolucency and emboli signals predict stroke in asymptomatic carotid stenosis," Neurology, vol. 77, pp. 751-758, 2011.

[13] A. AbuRahma, J. Wulu, and B. Crotty, "Carotid plaque ultrasonic heterogeneity and severity of stenosis," Stroke, vol. 33, pp. 1772-1775, 2002.

[14] A. L. Leahy, P. T. McCollum, T. M. Feeley, M. Sugrue, M. C. Grouden, D. J. O'Connell, D. J. Moore, and G. D. Shanik, "Duplex ultrasonography and selection of patients for carotid endarterectomy: Plaque morphology or luminal narrowing?," J. Vasc. Surg., vol. 8, pp. 558-562, 1998.

[15] M. Langsfeld, A. C. Gray-Weale, and R. J. Lusby, "The role of plaque morphology and diameter reduction in the development of new symptoms in asymptomatic carotid arteries," J. Vasc. Surg., vol. 9, pp. 548-557, 1989.

[16] A. C. Gray-Weale, J. C. Graham, J. R. Burnett, K. Byrne, and R. J. Lusby, "Carotid artery atheroma: Comparison of preoperative B-mode ultrasound appearance with carotid endarterectomy specimen pathology," J. Cardiovasc. Surg., vol. 29, pp. 676-681, 1988. 
[17] S. K. Kakkos, J. M. Stevens, A. N. Nicolaides, E. Kyriacou, C. S. Pattichis, G. Geroulakos, and D. Thomas, "Texture analysis of ultrasonic images of symptomatic carotid plaques can identify those plaques associated with ipsilateral embolic brain infarction," Eur. J. Vasc. Endovasc. Surg., vol. 33, no. 4, pp. 422-429, 2007.

[18] C. I. Christodoulou, C. S. Pattichis, M. Pantziaris, and A. Nicolaides, "Texture based classification of atherosclerotic carotid plaques," IEEE Trans. Med. Imag., vol. 22, no. 7, pp. 902-912, Jul. 2003.

[19] E. Kyriacou, M. Pattichis, C.S. Pattichis, A. Mavrommatis, C.I. Christodoulou, S. Kakkos, and A. Nicolaides, "Classification of atherosclerotic carotid plaques using morphological analysis on ultrasound images," J. Appl. Intell., vol. 30, no. 1, pp. 3-23, 2009.

[20] W. H. Press, S. A. Teukolsky, W. T. Vetterling, and B. P. Flannery, "Support Vector Machines," Numerical Recipes: The Art of Scientific Computing, 3rd. New York: Cambridge University Press, 2007, sec. 16.5.

[21] T. Joachims, Making Large-Scale SVM Learning Practical. Advances in Kernel Methods-Support Vector Learning, B. Schölkopf and C. Burges and A. Smola, Eds. Cambridge, MA: MIT Press, 1999, ch. 11.

[22] D. F. Specht, "Probabilistic neural networks," INNS Neural Netw., vol. 3, no. 1, pp. 109-118, 1990.

[23] M. Griffin, A. Nicolaides, and E. Kyriacou, "Normalisation of ultrasonic images of atherosclerotic plaques and reproducibility of grey scale median using dedicated software," Int. Angiol., vol. 26, pp. 372-377, 2007.

[24] A. Hartmann, J. Mohr, J. Thompson, O. Ramos, and H. Mast, "Interrater reliability of plaque morphology classification in patients with severe carotid artery stenosis," Acta Neurologica Scandinavica, vol. 99, pp. 6164, 1999.

[25] I. Momjian, S. Momjian, S. Albanese, M. Comelli, K. Lovblad, and R. Sztajzel, "Visual analysis or semi-automated gray-scale-based color mapping of the carotid plaque: Which method correlates the best with the presence of cerebrovascular symptoms and/or lesions on MRI?," J. Neuroimag., vol. 19, pp. 119-126, 2009.

[26] C. Petersen, P. Pecanha, L. Venneri, E. Pasanisi, L. Pratali, and E. Picano, "The impact of carotid plaque presence of morphology on mortality outcome in cardiological patient," Cardiovasc. Ultrasound, vol. 4, 2006.
[27] J. Dayhoff and J. DeLeo, "Artificial neural networks," Cancer, vol. 91, pp. 1615-1635, 2001.

[28] J. Tu, "Advantages and disadvantages of using artificial neural networks versus logistic-regression for predicting medical outcomes," J. Clin. Epidemiol., vol. 49, pp. 1225-1231, 1996.

[29] V. Vapnik, S. Golowich, and A. Smola, "Support vector method for function approximation, regression estimation, and signal processing," in Advances in Neural Information Process System., vol. 9. San Mateo, CA: Morgan Kaufmann, pp. 281-287, 1997.

[30] S. Li, J. Kwok, H. Zhu, and Y. Wang, "Texture classification using the support vector machines," Pattern Recog., vol. 36, pp. 2883-2893, 2003.

[31] A. Naylor, P. Gaines, and P. Rothwell, "Who benefits most from intervention for asympotmatic carotid stenosis: Patients or professionals?," Eur. J. Vasc. Endovasc. Surg., vol. 37, pp. 625-632, 2009.

[32] J. McPhee, A. Schanzer, L. Messina, and M. Eslami, "Carotid artery stenting has increased rates of post-procedure stroke, death and resource utilization than does carotid endarterectomy in the United States 2005," J. Vasc. Surg., vol. 48, pp. 1442-1450, 2008.

[33] M. Wholey, J. Barbato, and G. Al-Khoury, "Treatment of asymptomatic carotid disease with stenting," Semin. Vasc. Surg., vol. 21, pp. 95-99, 2008.

Authors' photographs and biographies not available at the time of publication. 